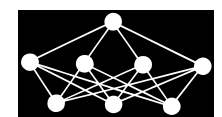

\title{
USING THE LISP-MINER SYSTEM FOR CREDIT RISK ASSESSMENT
}

\author{
P. Berka*
}

\begin{abstract}
Credit risk assessment, credit scoring and loan applications approval are one of the typical tasks that can be performed using machine learning or data mining techniques. From this viewpoint, loan applications evaluation is a classification task, in which the final decision can be either a crisp yes/no decision about the loan or a numeric score expressing the financial standing of the applicant. The knowledge to be used is inferred from data about past decisions. These data usually consist of both socio-demographic and economic characteristics of the applicant (e.g., age, income, and deposit), the characteristics of the loan, and the loan approval decision. A number of machine learning algorithms can be used for this purpose. In this paper we show how this task can be performed using the LISpMiner system, a tool that is under development at the University of Economics, Prague. LISp-Miner is primarily focused on mining for various types of association rules, but unlike "classical" association rules proposed by Agrawal, LISp-Miner introduces a greater variety of different types of relations between the left-hand and right-hand sides of a rule. Two other procedures that can be used for classification task are implemented in LISp-Miner as well. We describe the $4 \mathrm{ft}-$ Miner and KEX procedures and show how they can be used to analyze data related to loan applications. We also compare the results obtained using the presented algorithms with results from standard rule-learning methods.
\end{abstract}

Key words: data mining, decision rules, association rules, credit scoring

Received: May 4, 2015

DOI: $10.14311 / \mathrm{NNW} .2016 .26 .029$

Revised and accepted: October 26, 2016

\section{Introduction}

Credit risk refers to the risk that a borrower will default on any type of debt by failing to make required payments. To reduce the lender's credit risk, the lender may perform a credit check on the prospective borrower. The granting of credit then depends on the confidence the lender has in the borrower's credit worthiness. Most banks or lenders use some credit-scoring models (credit scorecards) to rank potential and existing customers according to their risk. One example is the FICO (Fair, Isaac and Company) score, the most popular credit score in the US. The FICO score is derived from positive and negative information in the

${ }^{*}$ Petr Berka, Dept. of Information and Knowledge Engineering, University of Economics, W. Churchill Sq. 4, CZ-130 67 Prague, Czech Republic, E-mail: berka@vse.cz 
credit record of a prospective borrower. This score considers the payment history (e.g., bankruptcy, late payments, or foreclosures), amounts owed (various info about debt), length of credit history (e.g., age of accounts), new credit (e.g., type of new credit) and types of credit used (e.g., consumer finance or mortgage). Each particular characteristic within every category is evaluated and added (in the form of a mathematical equation) to contribute to the final score. The five categories' proportions in the final score are: $35 \%$ takes the payment history, $30 \%$ takes the amounts owed, $15 \%$ takes the length of credit history, $10 \%$ takes the new credit and $10 \%$ takes the types of credit used. The FICO score ranges from 300 to 850 . The higher the score, the lower the risk. Another example is the Vantage score, which evaluates the loan applicant according to six categories: payment history (this category makes up $32 \%$ of the final score), usage of the available credit (23\%), total debt $(15 \%)$, past credits and credit history $(13 \%)$, number of past loan applications $(10 \%)$ and amount of credits on credit cards (7\%), The Vintage score ranges from 501 to 990 . Again, the higher the score, the better the rating. There are also some other scores that are used to evaluate the credit worthiness of a prospective loan applicant (see Tab. 1).

The main drawback of using these scores is the necessity to have available values for all input variables. Without this information, the weighted sum, that corresponds to a score cannot be computed. It is also reported, that the FICO score is not a good predictor in classifying between good loan applicants (who repay their loan) and bad applicants (who do not), as the average difference of FICO score between these two groups is very small.

\begin{tabular}{cccccc}
\hline Grade & FICO & Vantage & PLUS & TransUnion & CreditXpert \\
\hline A & $750-850$ & $901-990$ & $740-830$ & $845-925$ & $800-900$ \\
B & $700-749$ & $801-900$ & $695-739$ & $765-844$ & $740-799$ \\
C & $650-699$ & $701-800$ & $655-694$ & $685-764$ & $670-739$ \\
D & $600-649$ & $601-700$ & $590-654$ & $605-684$ & $610-669$ \\
F & $300-599$ & $501-600$ & $300-589$ & $150-604$ & $300-609$ \\
\hline
\end{tabular}

Tab. I Different credit scores (credit.com).

A credit score can be used as-is, can be turned into a grade (A-F) or fuzzy concepts (e.g., very poor, poor, fair, good, and excellent). Regardless of this representation, the final decision the bank makes is whether or not to approve a loan, i.e., to distinguish between "good" or "bad" applications. From this viewpoint, loan applications evaluation can be understood as a binary classification task. The knowledge to be used can be inferred from data about past decisions. These data usually consist off both socio-demographic and economic characteristics of the applicant (e.g., sex, income, and deposit), the characteristics of the loan, and the loan approval decision.

A number of machine learning algorithms can be used for this purpose. In this paper we show how this task can be solved using the LISp-Miner system, a tool that is under development at the University of Economics, Prague. The rest of the paper is organized as follows: Section 2 reviews related work on applying machine 
learning algorithms to credit risk assessment; Section 3 presents the LISp-Miner system; Section 4 describes the experiments carried out on data from the loan applications domain, and Section 5 concludes the paper.

\section{Related work}

Credit risk assessment, credit scoring and loan applications approvals are typical tasks that can be performed using machine learning or data mining techniques. Hence a lot of research has been carried out in this area. Chen et al. [7] use a two-stage approach composed of $k$-means clustering and support vector machines (SVM) classification together with computation of feature importance. $K$-means clustering is used to obtain homogeneous clusters of representative examples (isolated examples are eliminated, and inconsistent examples are re-labeled). The SVM classifier is then applied to these homogeneous clusters. Vinciotti and Hand [30] compare the use of the "classical" linear approach with the $k$-nearest-neighbor methods. They also discuss the issue of highly imbalanced classes (usually a large majority of applications are "good" ones) and describe four different scenarios how to handle this problem: the use of different weights for examples of different classes when classifying, the use of different weights for examples of different classes when building the model, the use of oversampling to balance the training data, and the use of separability criteria based on within class score distributions. Zhou and Wang [32] propose to use random forests to distinguish between good and bad loans if the classes are imbalanced. They use weighted random forests (here different weights of examples belonging to different classes are used during learning), and balanced random forests (here the data are oversampled). Lee, Chiu, Chou and $\mathrm{Lu}[20]$ propose to use the Classification and Regression Tree (CART) and Multivariate Adaptive Regression Splines (MARS) algorithms to classify clients of a local bank at Taiwan. Kim and Hwang [18] integrated multi-layer perceptron, discriminant analysis and decision tree models using genetic algorithms to create a system for credit risk evaluation. Galindo and Tamayo [13] presented a comparative analysis of different statistical and machine learning modeling methods of classification on mortgage loan data. Kotsiantis [19] proposed a selective voting method of representative machine learning algorithms (decision tree, neural network, Bayesian classifier, and $k$-NN classifier) to classify loan applications.

\section{The LISp-Miner system}

The LISp-Miner system is an academic data mining software tool developed at the University of Economics, Prague ${ }^{1}$, which is focused on mining various rule-like patterns from categorical data $[27,29]$. The system is a successor of the GUHA method, an original Czech approach to association rule mining from mid-1960s [15]. Contrary to "classical" association rules, GUHA (and LISp-Miner as well) introduce a greater variety of different types of relations between the left-hand (called antecedent) and right-hand (called succedent) sides of a rule and offers more expressive syntax for the rules.

\footnotetext{
${ }^{1}$ The system is freely available at http://lispminer.vse.cz.
} 
By now, LISp-Miner implements ten data mining procedures: 4ft-Miner (derived from the original GUHA procedure ASSOC), SD4ft-Miner, AC4ft-Miner, KLMiner, CF-Miner, SDKL-Miner, SDCF-Miner, KEX, ETree-Miner and MClusterMiner. Most of the procedures mine for various types of rule-like patterns - this makes LISp-Miner more focused on particular type of models than standard data mining tools. The procedures $4 \mathrm{ft}-$ Miner, SD4ft-Miner, AC4ft-Miner, KL-Miner, CF-Miner, SDKL-Miner, and SDCF-Miner mine for patterns which are to be interpreted by users and domain experts. They can then be used for data description, concept description or dependency analysis tasks ${ }^{2}$, KEX and ETree-Miner can be used for classification and the procedure MC-Miner that mines for clusters of examples can be used for segmentation. We will only describe in more detail the $4 \mathrm{ft}-$ Miner and KEX procedures, as they are used in our experiments. For description of the other procedures, see e.g., [27-29].

Most of the procedures implemented in LISp-Miner look for patterns (rules) that relate together so-called Boolean attributes. A Boolean attribute (also called a cedent) is a conjunction of partial cedents, a partial cedent is a conjunction or disjunction of literals and a literal is defined as $A($ coef $)$ or $\neg A($ coef $)$. Here $A$ is an attribute (variable) and coef (coefficient) defines a subset of possible values of A. So e.g., income(high), age(30-40,40-50) or $\neg$ city(Prague, Brno, Ostrava) are examples of literals. In these examples income (high) refers to a single value of the attribute income, age(30-40,40-50) refers to an interval created for two subsequent values of the discretized attribute age (numeric attributes must be discretized in advance, prior to using LISp-Miner data mining procedures - this can be done using the LISp-Miner module DataSource), and $\neg$ city(Prague, Brno, Ostrava) refers to a subset of values of the attribute city.

\subsection{Association rules and the 4ft-Miner procedure}

Association rules were proposed by R. Agrawal in the early 1990s as a tool for the so-called market basket analysis [1]. An association rule has the form of an implication

$$
X \Rightarrow Y,
$$

where $X$ and $Y$ are sets of items (itemsets) and $X \cap Y=\emptyset$. An association rule expresses that transactions containing items of set $X$ tend to contain items of set $Y$, so e.g. a rule

$$
\{A, B\} \Rightarrow\{C\}
$$

says that customers who buy products $A$ and $B$ also often buy product $C$. The two basic characteristics of an association rule are support and confidence. Support of an itemset $X$ is defined as the proportion of transactions in the data set which contain the itemset $X$. Confidence of an association rule $X \Rightarrow Y$ is defined as $\operatorname{support}(X \cup Y) / \operatorname{support}(X)$. This idea of association rules can be applied to any data in tabular, attribute-value form. So data describing values of attributes can be analyzed in order to find associations between conjunctions of attribute-value

\footnotetext{
${ }^{2}$ Refer to the typology of data mining tasks related to the CRISP-DM methodology [6].
} 
Berka P.: Using the LISp-Miner system for credit risk assessment

\begin{tabular}{cccc} 
Cond & Suc & $\neg$ Suc & $\sum$ \\
\hline Ant & $a$ & $b$ & $r$ \\
$\neg$ Ant & $c$ & $d$ & $s$ \\
\hline$\sum$ & $k$ & $l$ & $n$
\end{tabular}

Tab. II Four-fold contingency table created for examples satisfying condition $\gamma$.

pairs (categories). Let us denote these conjunctions as Ant (antecedent) and Suc (succedent) and the association rule as

$$
\text { Ant } \Rightarrow \text { Suc. }
$$

When using association rules for concept description, Suc will be a category of the target attribute.

The $4 \mathrm{ft}$-Miner procedure ${ }^{3}$ offers a more general form of the above-described association rules. $4 \mathrm{ft}-$ Miner mines for patterns (4ft-rules) of the form

$$
\text { Ant } \approx \text { Suc/Cond }
$$

where Ant, Suc and Cond (condition) are cedents, and $\approx$ (called quantifier) denotes a relationship between Ant and Suc for the examples from the analyzed data table that fulfill Cond. If the condition is empty then the procedure analyzes the whole data table. The relationships between Ant and Suc are defined (and evaluated) using frequencies from the four-fold contingency table as shown in Tab. 3.1; here $a$ denotes the number of examples that fulfill both $A n t$ and $S u c, b$ denotes the number of examples that fulfill Ant but not Suc, $c$ denotes the number of examples that fulfill $S u c$ but not Ant, and $d$ denotes the number of examples that fulfill neither Ant nor Suc. We sometimes use $r=a+b, k=a+c, s=c+d, l=b+d$ and $n=a+b+c+d$.

Examples of relationships between Ant and Suc as defined for the frequencies for the four-fold table, are a founded implication $\Rightarrow$ defined as

$$
\frac{a}{a+b},
$$

a founded equivalence $=$ defined as

$$
\frac{a+b}{a+b+c+d},
$$

or an $\chi^{2}$ chi-squared quantifier defined as

$$
\frac{(a d-b c)^{2}}{r k l s} n .
$$

The $4 \mathrm{ft}$-Miner procedure contains almost 20 different types of those relationships. Their description can be found e.g. in [27]

\footnotetext{
${ }^{3}$ This procedure extends the original GUHA procedure ASSOC from the mid. 1960s. The authors of 4ft-Miner as well as of most of the other procedures are Jan Rauch and Milan Šmůnek.
} 
Let us assume a toy loan application domain consisting of attributes income (having values low and high), balance (having values low, medium, and high), sex (having values male and female), unemployed (having values yes and no), and loan (having values yes and no). An example of a $4 \mathrm{ft}-\mathrm{rule}$ can be

$$
\text { balance }(\text { high,medium }) \wedge \neg \text { unemployed(yes }) \Rightarrow \operatorname{loan}(\text { yes }) / \text { sex(male })
$$

which says that in the group of men, if a person has a high or medium balance on his account and is not unemployed, then he will get the loan. 4ft-rules are thus much more expressive than "standard" association rules, where the only possible example is of the following type:

$$
\text { income }(\text { high }) \wedge \text { balance }(\text { high }) \Rightarrow \operatorname{loan}(\text { yes }) \text {. }
$$

When running an $4 \mathrm{ft}$ task, the user should specify the literals that can be used to compose Ant, Suc, and Cond, and the type and parameters that define the relation between Ant and Suc (e.g., formulas (5), (6), (7) with lower bounds on their values), and the lower bound for frequency $a$ from the four-fold contingency table.

Unlike the well-known apriori algorithm, where single itemsets (i.e., conjunctions of categories) are generated in the first step and then each itemset (conjunction) is divided into antecedent and succedent [1], in 4ft-Miner each part of a rule (i.e., antecedent, succedent, and eventually condition) is generated separately, so we can easily set the target attribute as the only one that can occur in the succedents. Moreover, we can control the complexity of the searched rule space by determining (using parameters maxlen $A$, maxlen $S$ and maxlenC) the maximum number of literals that can occur in cedents of each respective part of a rule.

The result of the analysis using 4ft-Miner is usually a huge set of rules that should be inspected and evaluated by the domain experts and users (see Fig. 2). Let us stress here that the quantitative characteristics of the found rules do not guarantee usefulness of the rules. We can find a lot of rules with high confidence (especially those with low support), that do not represent any reasonable knowledge.

\subsection{Decision rules and the KEX procedure}

Decision rule in the form

$$
\text { Ant } \Rightarrow C
$$

where Ant (antecedent, condition) is a conjunction of values of input attributes (called categories or selectors) and $C$ is a category of class attribute $C$, are one of the most popular formalisms of how to express classification models learned from data. The commonly used approach to learning decision rules is the set covering approach also called "separate and conquer". The basic idea of this approach is to create a rule that covers some examples of a given class, and remove these examples from the training set (see Algorithm 1). This is repeated for all examples not covered so far. The other way to create decision rules is the compositional approach. In this approach the covered examples are not removed during learning, 
so an example can be covered with more rules. Thus more rules can be used during classification. In the compositional approach, all applicable rules are used and their particular contributions to classification are combined into the final decision. To do this, a certain numerical value is usually added to the rule. The KEX algorithm reported in this paper fits into the second-described type of methods [3].

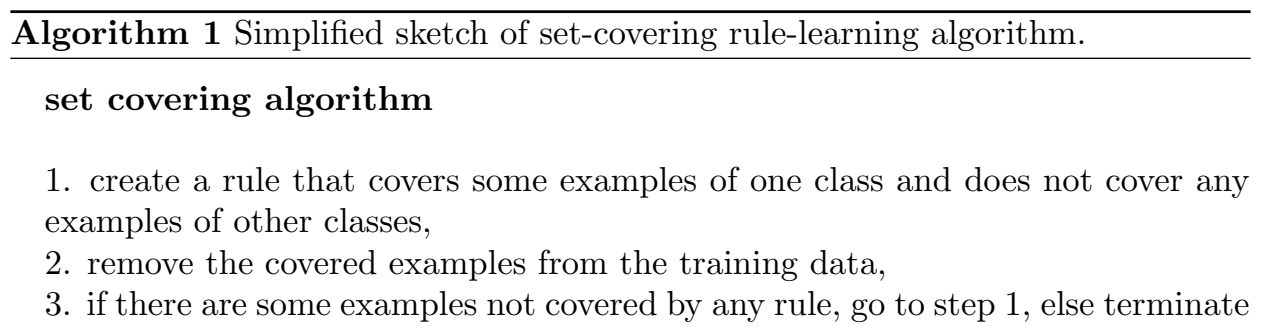

The KEX algorithm [3] learns weighted decision rules in the form

$$
\text { Ant } \Rightarrow C(w),
$$

where Ant (antecedent, condition) is a conjunction of values of input attributes (called categories), $C$ is the class attribute, and weight $w \in[0,1]$ expresses the uncertainty of the rule. Algorithm 2 shows a simplified version of the algorithm. KEX works in an iterative way, testing and expanding an implication $A n t \Rightarrow C$ in each iteration. This process starts with "empty rule" with the weight equal to the relative frequency of $C$ in data and stops after evaluating all implications which were created according to the user-defined criteria with a maximum length of Ant. $(\operatorname{lmax})$, minimum frequency of Ant, $(\mathrm{fmin})$ and minimum validity of $A n t \Rightarrow C$ $(P \min )$. The implications are evaluated according to decreasing frequency of Ant. When evaluating an implication, its validity (conditional probability $P(C / A n t)$ ) is computed. If this validity statistically differs from the composed weight (value obtained when composing weights of all sub-rules of the implication Ant $\Rightarrow C$ ) significantly, then this implication is added to the knowledge base. To test the difference between the validity and the composed weight, we use the chi-square goodness-of-fit test. The weight of the newly added rule is computed from the validity and from the composed weight using the inverse composing function [14]. For composing weights $w_{1}$ and $w_{2}$ we use a pseudo-Bayesian (Prospector-like) combination function [9]

$$
w_{1} \oplus w_{2}=\frac{w_{1} \cdot w_{2}}{w_{1} \cdot w_{2}+\left(1-w_{1}\right) \cdot\left(1-w_{2}\right)} .
$$

During expansion, new implications are created by adding single categories to Ant. These categories are added in descending order of their frequencies. New implications are stored (according to frequencies of $A n t$ ) in an ordered list of implications. So KEX generates every implication only once and for any implication in question all its sub-implications have already been tested.

When using the set of rules created by KEX for classification of an example, all applicable rules are found and their weights are combined using Eq. (12) to 


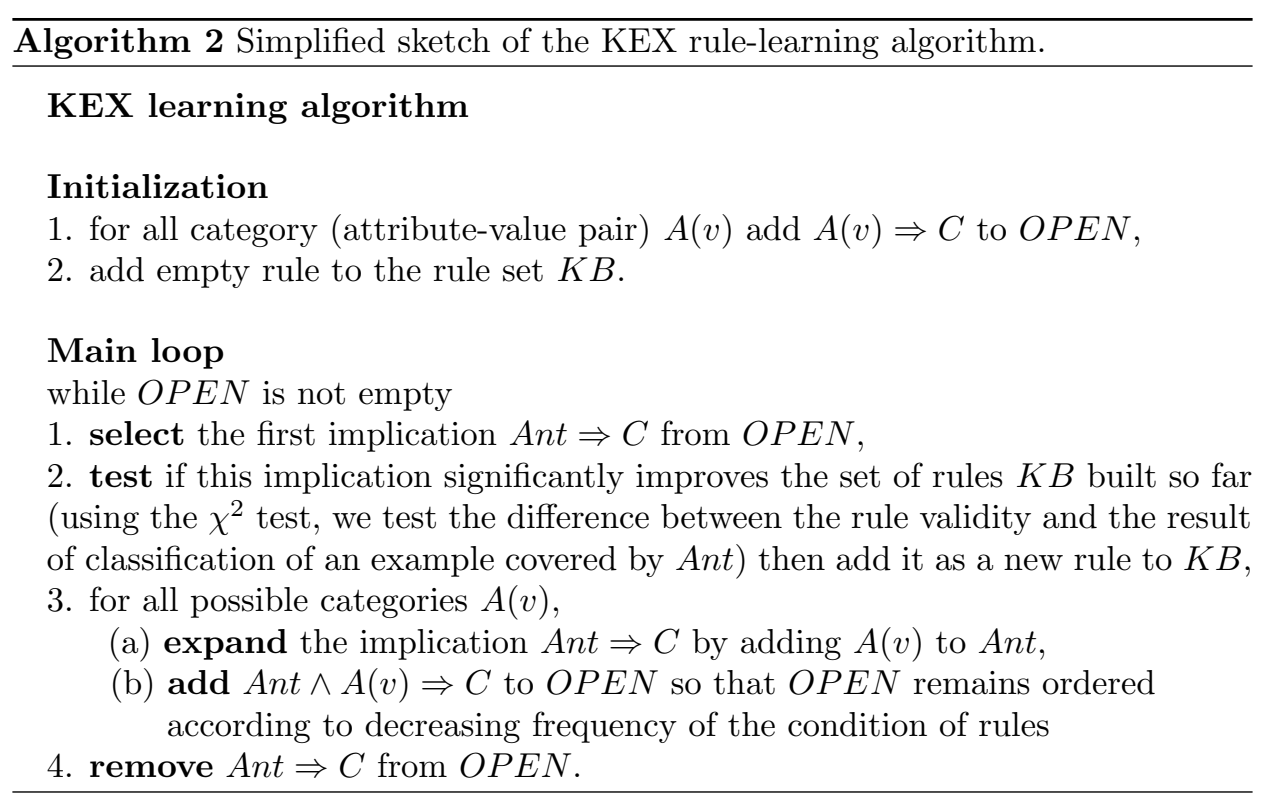

compute composed weights for each class. The classified example is then assigned to the class with the highest composed weight. For binary classification problems, we usually create rules towards only one of the classes. Thus the composed weight close to 1 is interpreted as classification of the given example into this class while the composed weight close to 0 is interpreted as classification of the given example into the opposite class. We can use these weights in the following decision strategy:

- if the composed weight $>\alpha$, then the example belongs to the class,

- if the composed weight $<(1-\alpha)$, then the example belongs to the opposite,

- if the composed weight is in the interval $[1-\alpha, \alpha]$, then we do not classify.

Here $\alpha$ is a threshold that can be set by the user. We will show in Section 4 the impact of the value $\alpha$ on the classification results in our case study. Generally speaking, when increasing the value of $\alpha$, the percentage of correctly classified examples usually increases (the ratio behind this observation is that the higher the weight of a class, the more likely the classification result will be correct), but the number of examples for which the model makes a decision always decreases. This strategy allows us to build a decision support system that will decide only for "easy-to-decide" situations and leaves the more complicated cases to the human expert.

As stated earlier in Section 3, LISp-Miner can work only with categorical attributes, so numeric attributes must be discretized in advance. In addition to two standard discretization methods, equidistant and equifrequent discretization, numeric attributes can be also discretized in a "class-sensitive" way. Here the intervals are created in such a way that the discretized attribute can be used for classification. This means the resulting intervals should contain a majority of examples 
belonging to one class. There is a number of such class-sensitive discretization algorithms $[10,17,21]$. In our experiments reported in Section 4 we used a discretization algorithm closely related to KEX [4]. Algorithm 3 shows a simplified sketch of this algorithm. The algorithm starts by creating initial intervals for each value of the numeric attribute that occurs in the data and then merges the neighboring intervals if they share the same qualitative distribution of examples into classes (i.e., they share the same majority class).

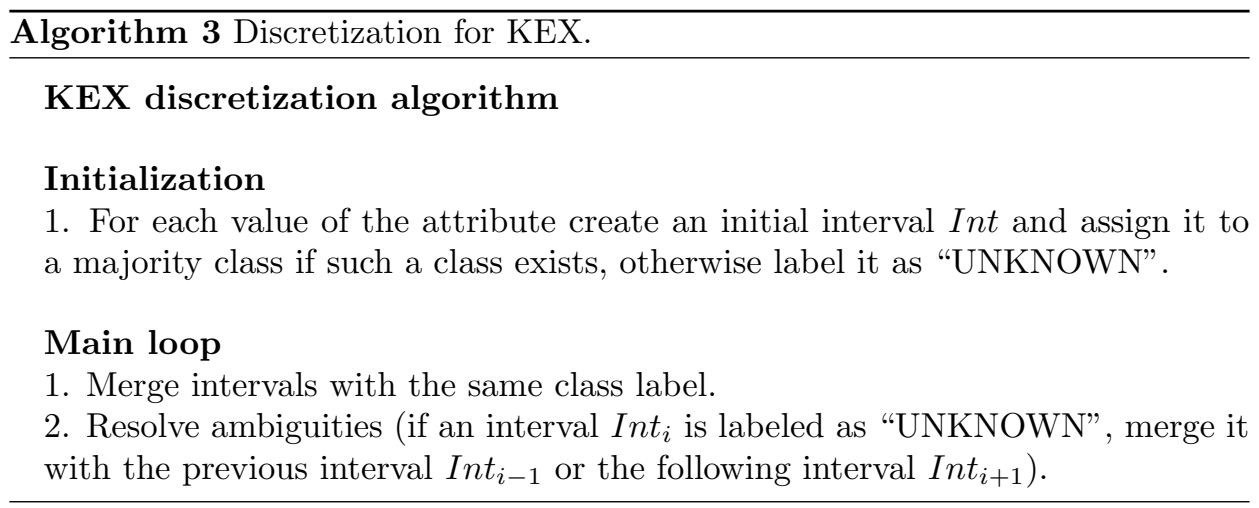

\section{Experiments with the loan application data}

We used the 4ft-Miner and KEX procedures to analyze several data sets from the credit risk assessment domain. The first three data sets are taken from the UCI Machine Learning Repository that contains a number of reference data sets used by the machine learning community to evaluate various machine learning and data mining algorithms (www.ics.uci.edu/ mlearn/MLRepository.html). Australian credit data is used for credit card applications. The dataset contains a mixture of continuous, nominal with small numbers of values, and nominal with larger numbers of values attributes. All attribute names and values have been changed to meaningless symbols to protect confidentiality of the data. This data set was first used by Quinlan in 1987 [23]. German credit data were provided by prof. Hofmann from the University of Hamburg; this data consists of 7 numerical and 13 categorical input attributes. Japan Credit Data set represents consumer loans for a specific purpose (e.g., car, or a PC); this data set was prepared by Chiharu Sano in 1992. The Discovery Challenge data set was prepared for the Discovery Challenge workshop held at the European Conference of Principles and Practice on Knowledge Discovery PKDD1999 [2], the analyzed table (6181 examples, 7 input attributes) is an excerpt from the whole database used in the workshop.

Tab. III shows the basic characteristics of the used data (number of examples, number of attributes, number of classes, and the default accuracy computed as the percentage of the majority class). The last column, maximal accuracy, reflects the 
Neural Network World 5/2016, 497-518

\begin{tabular}{cccccc}
\hline Data & examples & attributes & classes & default acc. & maximal acc. \\
\hline ACred & 690 & 15 & 2 & 0.56 & 0.99 \\
GCred & 1000 & 20 & 2 & 0.70 & 1.00 \\
JCred & 125 & 10 & 2 & 0.68 & 1.00 \\
DChall & 6181 & 7 & 2 & 0.88 & 1.00 \\
\hline
\end{tabular}

Tab. III Basic characteristics of the used data sets.

amount of noise in the data ${ }^{4}$. Some data sets contain only categorical attributes; these data sets can be used directly without pre-processing. Other data sets contain numeric attributes. In this case, the data must be discretized prior to the use of the learning algorithms.

In our experiments we compared the results obtained with the two procedures described in the previous subsections (4ft-Miner and KEX) with the results obtained when using standard rule-learning algorithms as implemented in Weka, a well-known free data mining suite from University Waikato, New Zealand [16].

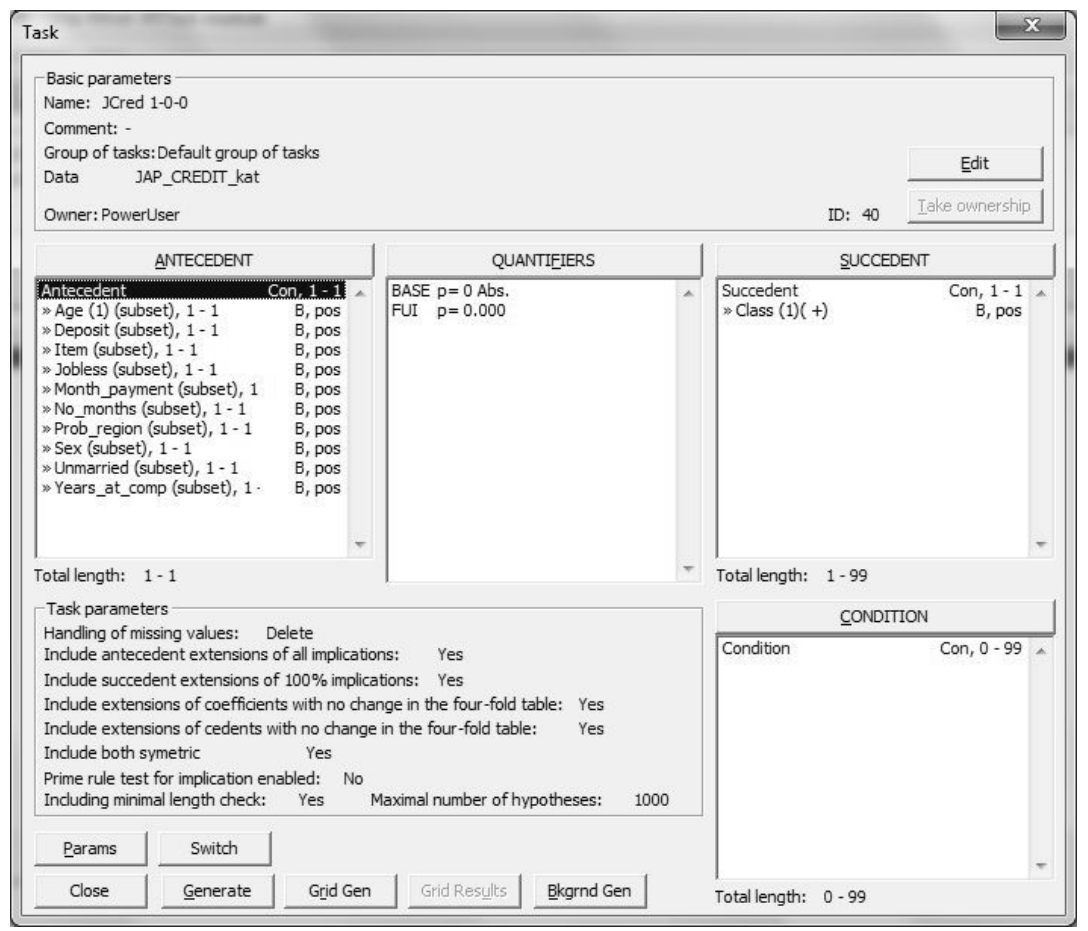

Fig. 1 Screenshot with input setting for 4ft-Miner for Japan Credit Data.

\footnotetext{
${ }^{4}$ We refer by noise to situations when examples with the same values of input attributes belong to different classes. Such examples are undistinguishable and thus increase the classification error. If the maximal accuracy equals 1 , then the data are noise free.
} 
In the first experiment, we applied 4ft-Miner to find strong interesting relationships between input attributes and class; we can view such relationships as concept descriptions of the respective classes. While strong relations can be found automatically (in our experiment by setting the founded implication $p=0.9$ ), what is interesting should be determined by the domain expert. The screenshot in Fig. 1 shows the input settings of this experiment for Japan Credit Data and the screenshot in Fig. 2 shows the corresponding results. Tab. 4 shows some of the strong rules found in the Japan Credit Data. Here high confidence and high support identifies a (relatively) large group of good loan applicants, in our case men and/or older persons working for the same company for several years (rules 7 and 10).

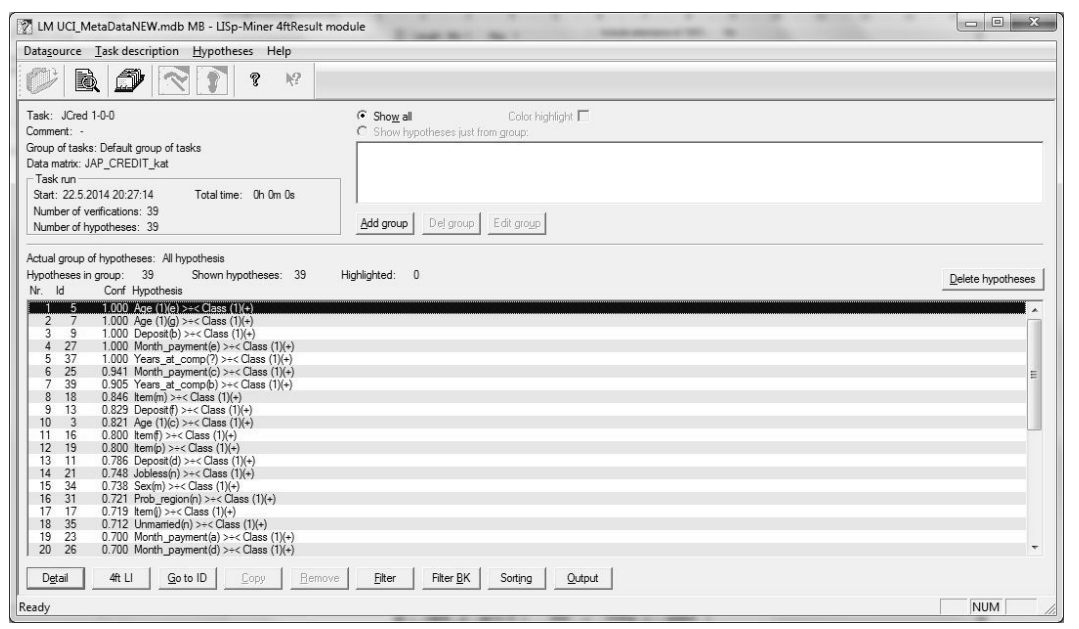

Fig. 2 Screenshot with example results of $4 \mathrm{ft}$-Miner for Japan Credit Data.

\begin{tabular}{llcc}
\hline no. & Hypothesis & Conf & Supp \\
\hline 01: & Age $(c)$ \& Month_payment $(\mathrm{c}) \Rightarrow$ Class $(+)$ & 1.00 & 0.09 \\
02: & Item(j) \& Years_at_comp $(\mathrm{b}) \Rightarrow$ Class $(+)$ & 1.00 & 0.14 \\
03: & Jobless(n) \& Month_payment $(\mathrm{c}) \Rightarrow$ Class $(+)$ & 1.00 & 0.13 \\
04: & Month_payment(c) \& No_months(b) $\Rightarrow$ Class $(+)$ & 1.00 & 0.10 \\
05: & Month_payment(c) \& Unmarried $(\mathrm{n}) \Rightarrow$ Class $(+)$ & 1.00 & 0.09 \\
06: & Month_payment(c) \& Years_at_comp $(\mathrm{b}) \Rightarrow$ Class $(+)$ & 1.00 & 0.09 \\
07: & Sex(m) \& Years_at_comp(b) $\Rightarrow$ Class $(+)$ & 0.97 & 0.27 \\
08: & Unmarried(y) \& Years_at_comp $(\mathrm{b}) \Rightarrow$ Class $(+)$ & 0.96 & 0.18 \\
09: & Deposit(f) \& Years_at_comp $(\mathrm{b}) \Rightarrow$ Class $(+)$ & 0.95 & 0.16 \\
10: & Age $(c) \&$ Years_at_comp $(b) \Rightarrow$ Class $(+)$ & 0.95 & 0.30 \\
\hline
\end{tabular}

Tab. IV Strong association rules found by 4ft-Miner for Japan Credit Data.

We also compared between 4ft-Miner and KEX. The idea of this comparison was to have a look at the "reduction rate" that KEX applies to the implications (association rules) when deciding if an implication should be added to the KB or 
not. So the parameter settings were the same for both $4 \mathrm{ft}-\mathrm{Miner}$ and KEX. We used two different settings (scenarios) which were derived from recommended strategies for KEX and that will also be applied in the second experiment. In the first setting KEX1 or 4FT1 (in KEX called "minimal analysis" strategy) we give no restriction on the values of frequency and validity $(\operatorname{lmax}=1, f \min =0 \%$ and $\operatorname{Pmin}=0)$, in the second setting KEX2 or 4FT2 (in KEX called "strong analysis" strategy) we are interested only in the implications with high validity $(\operatorname{lmax}=2, f \min =1 \%$ and Pmin = 0.9). The screenshot in Fig. 3 shows the input settings for KEX1 for Japan Credit Data and the screenshot in Fig. 4 shows the corresponding results. Tab. ?? shows (for both settings) the numbers of rules created by $4 \mathrm{ft}-$ Miner and KEX (columns "4FT1", "KEX1" for the first setting and columns "4FT2", "KEX2" for the second setting) and the reduction ratio computed as the number of KEX rules divided by the number of $4 \mathrm{FT}$ rules (column "reduction").

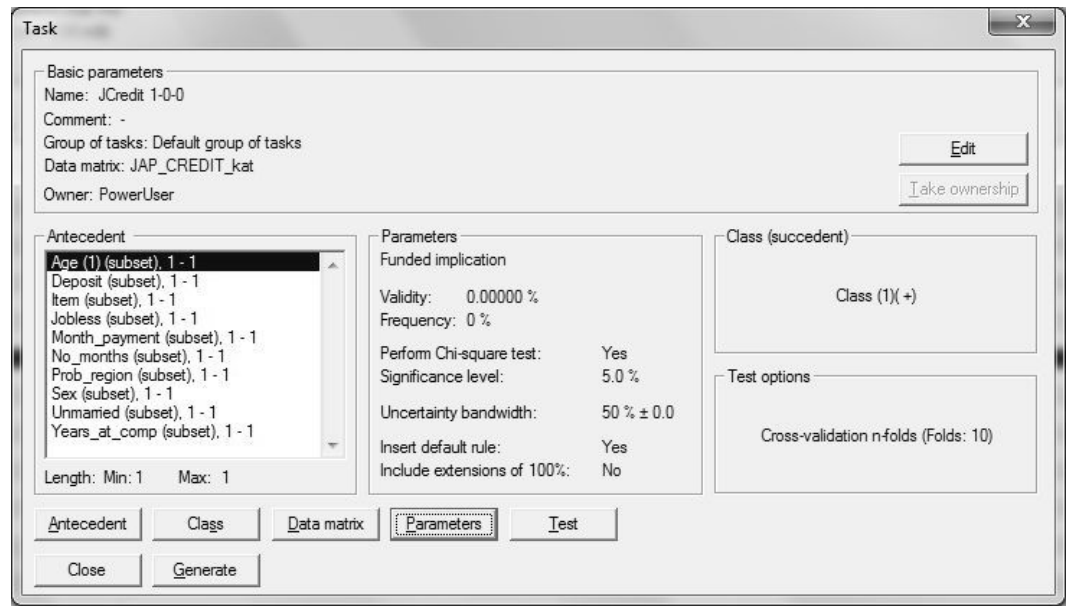

Fig. 3 Screenshot with input setting for KEX for Japan Credit Data.

\begin{tabular}{ccccccc}
\hline & \multicolumn{3}{c}{ Setting 1 } & \multicolumn{3}{c}{ Setting 2 } \\
Data & KEX1 & 4FT1 & reduction & KEX2 & 4FT2 & reduction \\
\hline ACred & 36 & 57 & 0.63 & 60 & 174 & 0.34 \\
JCred & 11 & 39 & 0.28 & 25 & 118 & 0.21 \\
GCred & 27 & 79 & 0.34 & 95 & 279 & 0.34 \\
DChall & 17 & 36 & 0.47 & 104 & 230 & 0.45 \\
\hline
\end{tabular}

Tab. V Quantitative comparison of results from $4 \mathrm{ft}$-Miner and $K E X$.

In the second experiment, we compared KEX with the rule-learning algorithms PRISM, Jrip, PART and Ridor implemented in Weka.

PRISM is a set-covering algorithm that produces rules by rule specializations starting from a rule with an empty antecedent and stops adding conditions when the rule reaches the accuracy equal to 1 [5]. The algorithm thus assumes that the 


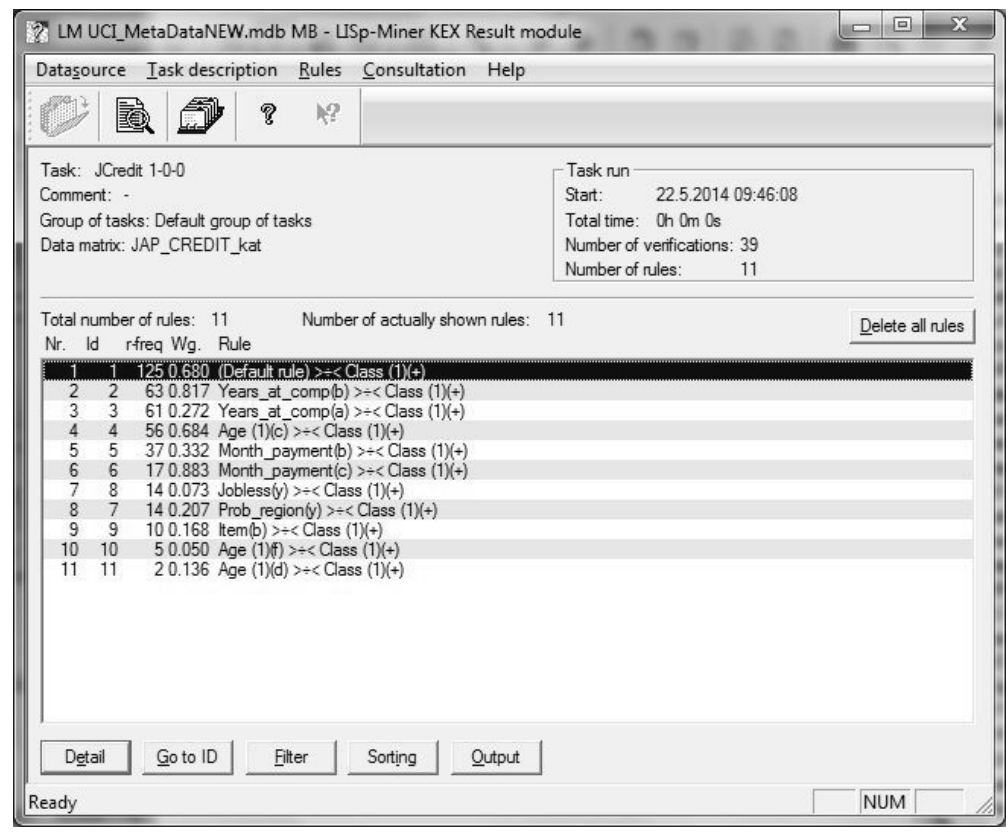

Fig. 4 Screenshot with example results of KEX for Japan Credit Data.

data are noise-free. PRISM was inspired by the decision tree learning algorithm ID3 [22]. But unlike ID3, where the best splitting attribute is found, the best attribute-value pair (category) is identified in PRISM. To do this, the information gain is computed using

$$
I(A(v), C)=\log _{2}\left(\frac{P(C \mid A(v))}{P(C)}\right)
$$

and the category $A(v)$ with the highest information gain is selected ${ }^{5}$.

As $P(C)$ is constant for different $A(v)$ and binary logarithm is an increasing function, it is sufficient to select the best category by maximizing $P(C \mid A(v))$. The "create a single rule" part of the generic set-covering algorithm shown in Algorithm 1 thus has the form shown in Algorithm 4.

Jrip is an implementation of the RIPPER (Repeated Incremental Pruning to Produce Error Reduction) algorithm. This algorithm is again a variant of the setcovering approach. It performs rule induction (top-down specialization of rules) followed by a post-processing step that prunes the rules to improve the classification accuracy [8]. The algorithm was intended to learn rules from large noisy data. Jrip repeatedly creates a rule on a growing set and prunes it on a pruning set. A rule is created in a top-down manner, adding a category $A(v)$ or a condition $A \leq \theta$ or $A \geq \theta$ (Jrip can thus directly work with numeric attributes). The best rule specialization is selected by maximizing the information gain adopted from the ILP rule-learning system FOIL [24]. The "create a single rule" part of the algorithm is shown in Algorithm 5.

\footnotetext{
${ }^{5}$ We use the same notation as in our description of KEX in Section 3.2
} 


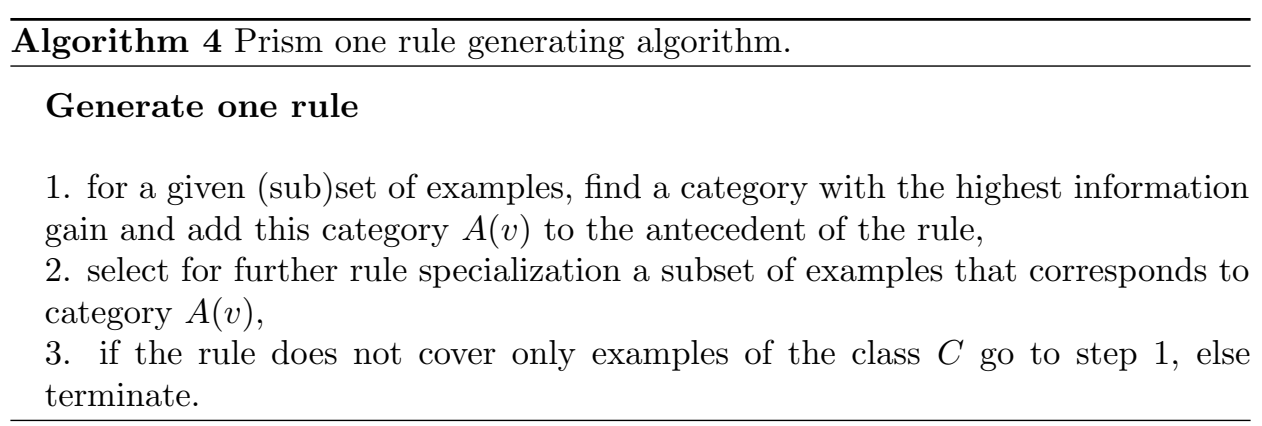

Algorithm 5 Simplified sketch of Jrip rule-generating part.

\section{Generate one rule}

split uncovered examples into a growing set and a pruning set on growing set do

1. add $A(v)$ or $A \leq \theta$ or $A \geq \theta$ that maximizes the information gain to the Ant of the rule,

2. if rule covers some negative examples, go to step 1, else terminate,

on pruning set do

3. remove a final sequence of conditions from Ant to maximize the ratio $(p-$ $n) /(p+n)$, where $p$ denotes the number positive examples of the class covered by the rule and $n$ is the number of negative examples of the class covered by the rule.

PART is a set-covering algorithm based on partial decision trees. To make a single rule, a pruned decision tree is built for the current (sub)set of examples and the path to the leaf with the largest coverage is turned into a rule [11]. To reduce the computational time of the algorithm (a naive implementation will create and prune a full tree for every rule), partial decision trees are created during the rule creation process instead. A partial decision tree is a tree that contains branches to undefined subtrees, i.e. contain nodes that were not expanded. Algorithm 6 shows the algorithm for creating partial trees. The way how a splitting attribute is selected and how the tree is pruned is the same as used in the C4.5 algorithm [25].

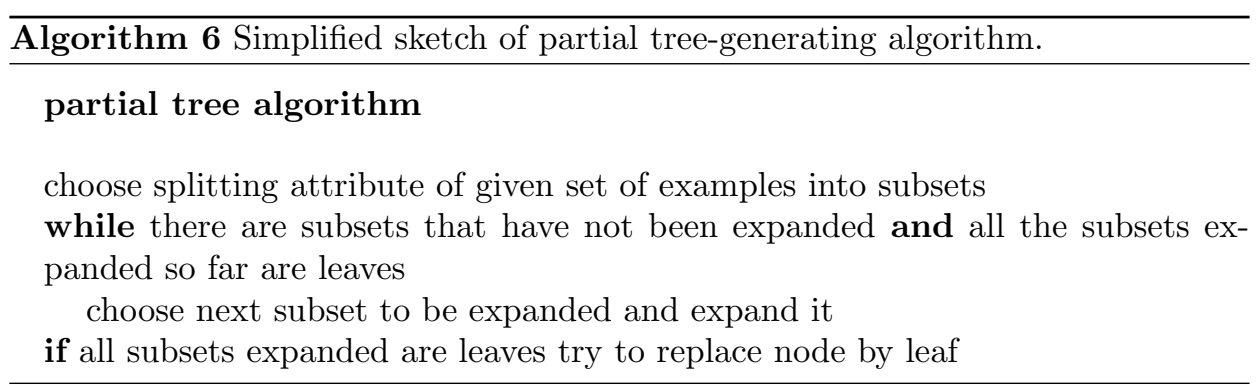


The Ridor (RIpple DOwn Rules) algorithm learns rules in noisy domains, i.e., rules that need not to have $100 \%$ classification accuracy. This algorithm recursively generates (by rule specialization) if-true and if-false rules [12]. The ripple down rules form a binary tree, where each node corresponds to an antecedent and the branches correspond to true/false values. If a parent rule is activated (i.e., its antecedent is true) then its child if-true rule is checked. If the child if-true rule is activated, then the class assigned to the example will correspond to the class of this rule; otherwise the class assigned to the example will correspond to the class of the parent rule. If the parent rule is not activated, its if-false child rule is checked. If the child if-false rule is activated, then the class assigned to the example will correspond to the class of this rule. The algorithm for generating a single rule is inspired by the PRISM algorithm [5], but the stopping condition for rule specialization is different. Algorithm 7 shows its steps, a formula

$$
r=\sum_{i=c}^{s}\left(\begin{array}{c}
s \\
i
\end{array}\right) p^{i}(1-p)^{s-i}
$$

is used to assess the quality of a category or of a rule ( $r$ should be maximized). Here $p$ stands for the relative frequency of the class, $s$ stands for the number of examples covered by the category or rule, and $c$ stands for the number of examples that are covered and belong to the class. To create the ripple-down structure, this algorithm is recursively called twice. The if-true child rule is created for the next most frequent class of the examples covered by the parent rule. The if-false child rule is created for the same class as the parent rule but the computation is carried out for the examples not covered by the parent rule.

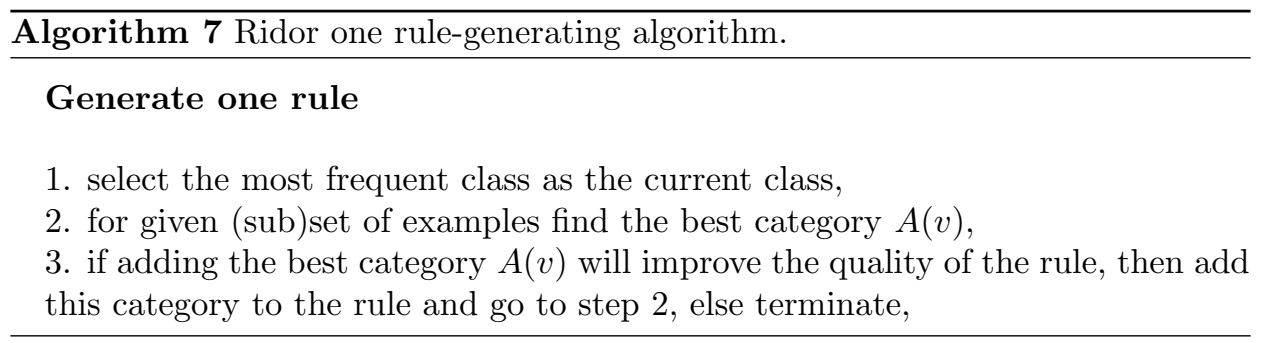

Tab. VI summarizes the achieved results. The table shows for each of the used learning algorithms the number of rules and the classification accuracy reached using a 10-fold cross-validation test. We used standard settings for PRISM, Jrip, PART and Ridor and two different settings for KEX. Here, KEX1 and KEX2 are defined in the same way as when comparing KEX and 4ft-Miner. Numbers in bold print denote for each data set the best results in terms of classification accuracy and size of the rule set. We compute an overall rank as a weighted sum

$$
2 \times \text { acc.rank }+ \text { rul.rank. }
$$

The "winning" algorithm is the algorithm that minimizes this sum. So, e.g., for Japan Credit Data, KEX2 was the best in accuracy and fifth in rule-set size (which gives the overall rank of $2 \times 1+5=7$ ), and PRISM was third in accuracy and 
first in rule set size (which gives the overall rank $2 \times 3+1=7$ ); 7 was the smallest value of the overall rank achieved for this data. The table suggests that PART was outperformed by the other algorithms (PART never became a winner), and that the other algorithms are comparable. But no general conclusions about the superiority of an algorithm can be drawn from the experiments. Actually, the well-known no-free-lunch theorem gives an evidence that there is no single "best" machine learning algorithm regardless on the analyzed data [31].

\begin{tabular}{ccccccc}
\hline Data & $\begin{array}{c}\text { KEX1 } \\
\text { rul./acc. }\end{array}$ & $\begin{array}{c}\text { KEX2 } \\
\text { rul./acc. }\end{array}$ & $\begin{array}{c}\text { PART } \\
\text { rul./acc. }\end{array}$ & $\begin{array}{c}\text { PRISM } \\
\text { rul./acc. }\end{array}$ & $\begin{array}{c}\text { Jrip } \\
\text { rul./acc. }\end{array}$ & $\begin{array}{c}\text { Ridor } \\
\text { rul./acc. }\end{array}$ \\
\hline ACred & $36 / 0.86$ & $60 / 0.83$ & $29 / 0.85$ & $197 / 0.76$ & $\mathbf{5 / 0 . 8 5}$ & $6 / 0.85$ \\
JCred & $11 / 0.76$ & $\mathbf{2 5 / 0 . 7 9}$ & $7 / 0.72$ & $\mathbf{4} / \mathbf{0 . 7 4}$ & $5 / 0.71$ & $4 / 0.70$ \\
GCred & $\mathbf{2 7 / 0 . 7 3}$ & $95 / 0.71$ & $68 / 0.70$ & $362 / 0.66$ & $8 / 0.69$ & $\mathbf{2 / 0 . 7 1}$ \\
DChall & $17 / 0.88$ & $104 / 0.88$ & $134 / 0.98$ & $\mathbf{2 0 3 / 0 . 9 9}$ & $\mathbf{4 1 / 0 . 9 6}$ & $73 / 0.92$ \\
\hline
\end{tabular}

Tab. VI Classification results of KEX and other rule learning algorithms.

The results shown in Tab. VI present the classification accuracy of KEX for the value of the threshold $\alpha$ set to 0.5 . For this setting, all examples for which the composed weight of the class differs from 0.5 will be classified. Figs. $5-8$ show the effect of different values of $\alpha$ on the classification accuracy and the ratio of classified examples (out of all examples). In all graphs the x-axis gives the values of $\alpha$, and the y-axis gives the classification accuracy or the number of classified examples respectively. The graphs in Figs. 5 and 6 show the results for the "minimal analysis" strategy and graphs in Figs. 7 and 8 show the results for the "strong analysis" strategy. We can conclude from these graphs that

- as we expected, when increasing the value of $\alpha$, the classification accuracy (i.e., the relative number of correctly classified examples out of all classified examples) increases and the relative number of classified examples (out of all examples) decreases;

- when increasing the value of $\alpha$, the improvement of classification accuracy was slightly better for the minimal analysis strategy, but at the same time the decrease of the number of classified examples for the minimal analysis strategy was significantly higher. This seems to indicate that the results for strong analysis are more robust.

We present the source of the difference between the results of KEX1 and KEX2 settings for Japan Credit Data in Figs. 9 and 10. Both graphs have the class indicator values on the $\mathrm{x}$-axis (to be able to better display the graph we jitter the values of class 1 in the range 0.8 to 1.2 and the values of class -1 in a range from -1.2 to -0.8$)$ and the composed weight on the y-axis.

A perfect rule base created by KEX would assign weights greater than 0.5 to examples of class 1 and weights smaller than 0.5 to examples of class -1 . So correctly classified examples are (in both Figures) indicated by the rectangles. Since the composed weights are closer to the extreme values 0 and 1 for the KEX2 
Berka P.: Using the LISp-Miner system for credit risk assessment

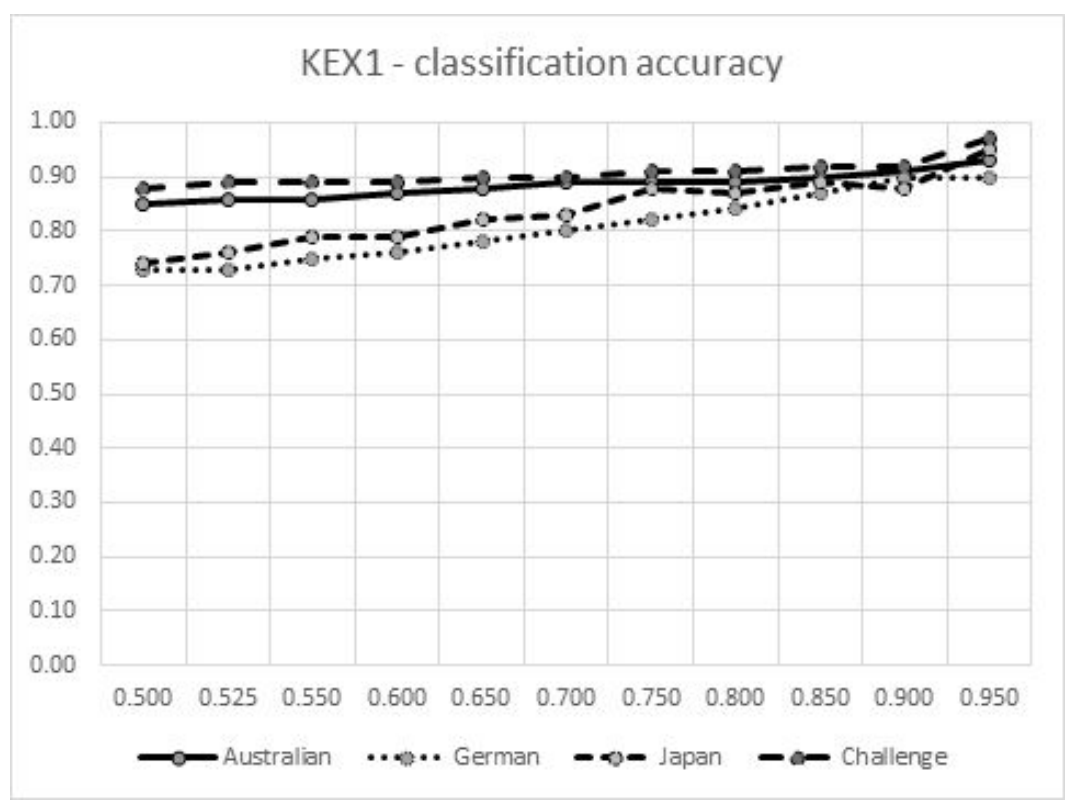

Fig. 5 The impact of $\alpha$ on the classification accuracy, KEX1 setting.

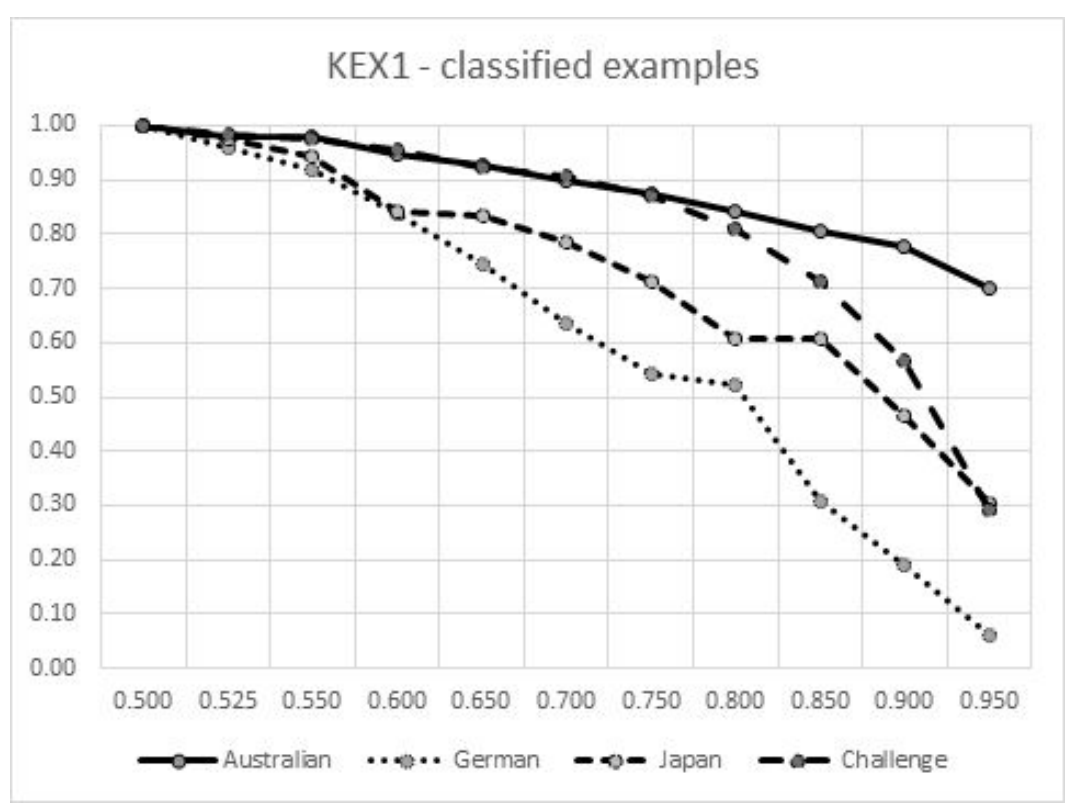

Fig. 6 The impact of $\alpha$ on the number of classified examples, KEX1 setting.

setting, when increasing the value of $\alpha$ we will be able to classify more examples than for the KEX1 setting. We can also see that when using the KEX2 setting, no example of class 1 was misclassified as an example of class -1 . 
Neural Network World 5/2016, 497-518

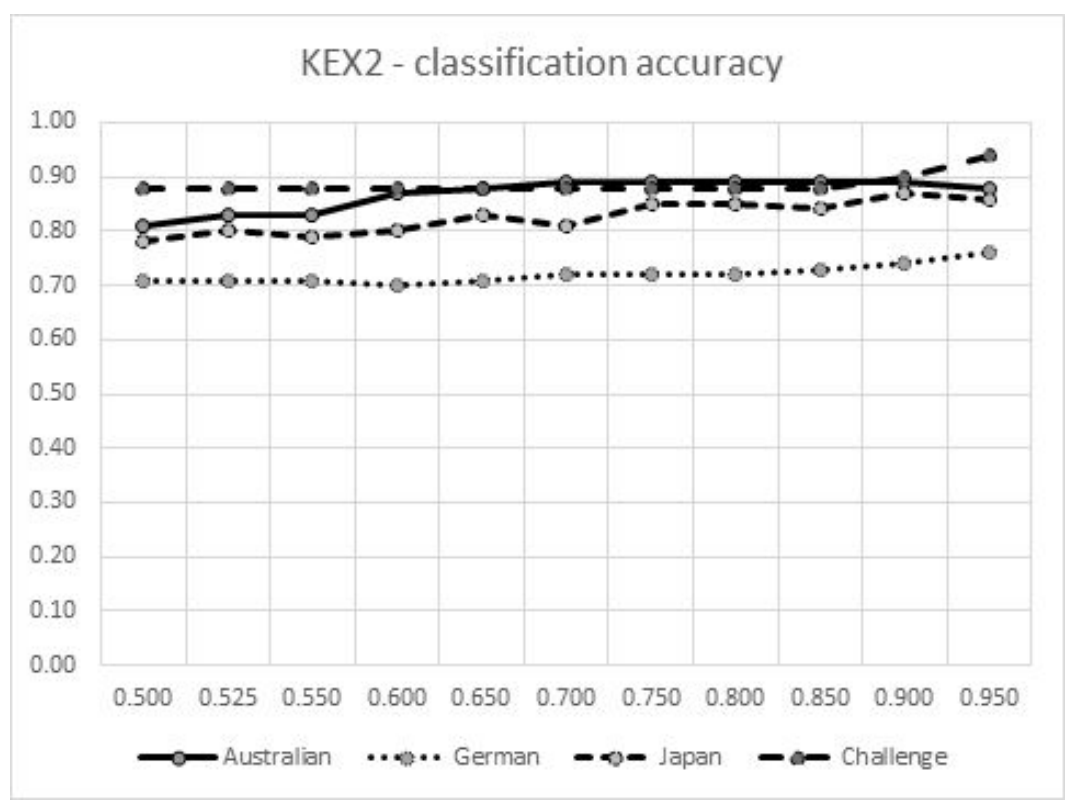

Fig. 7 The impact of $\alpha$ on the classification accuracy, KEX2 setting.

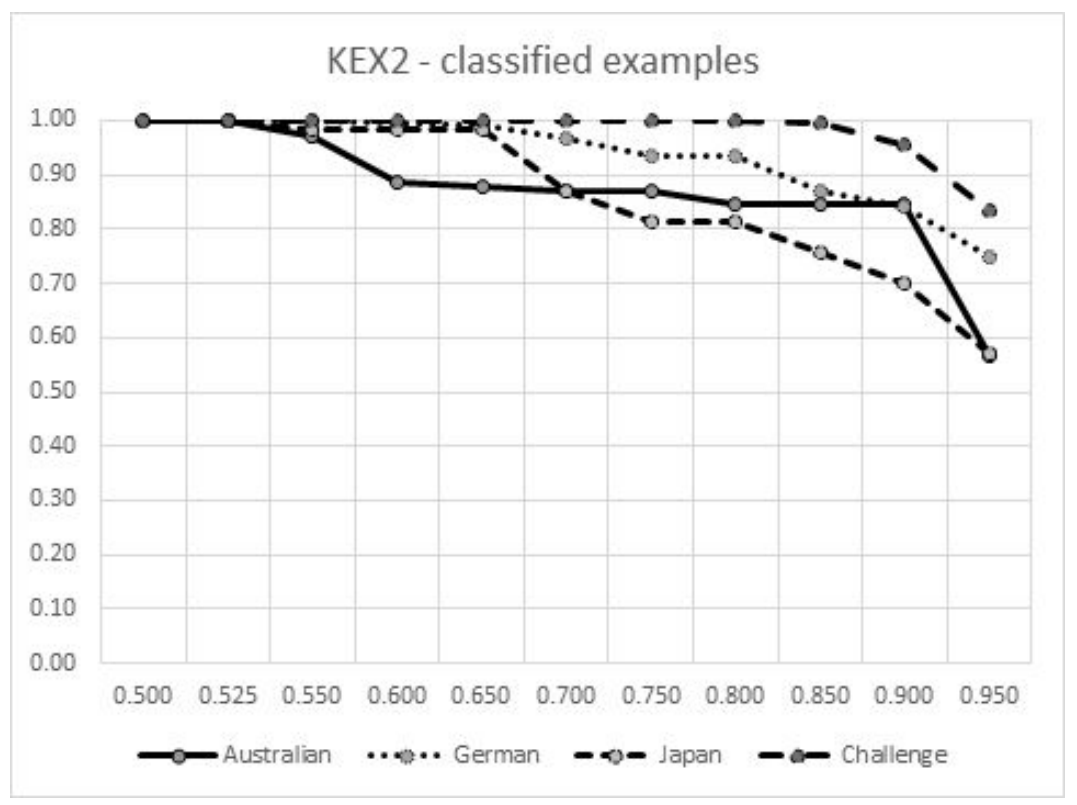

Fig. 8 The impact of $\alpha$ on the number of classified examples, KEX2 setting. 
Berka P.: Using the LISp-Miner system for credit risk assessment

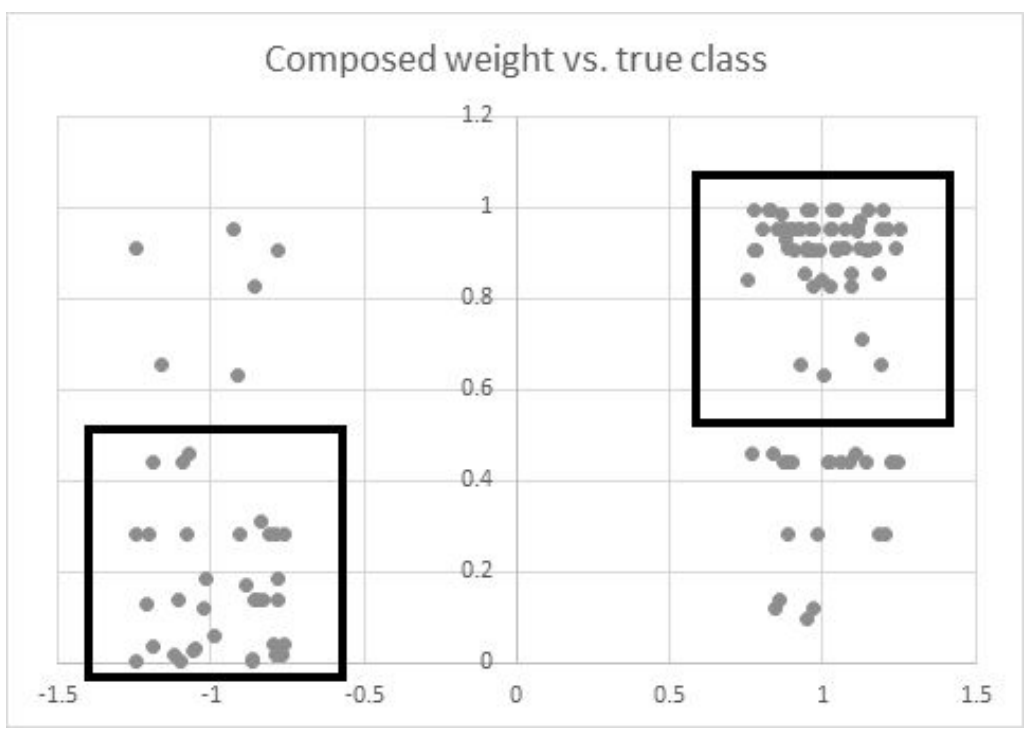

Fig. 9 Composed weights assigned to examples, KEX1 setting.

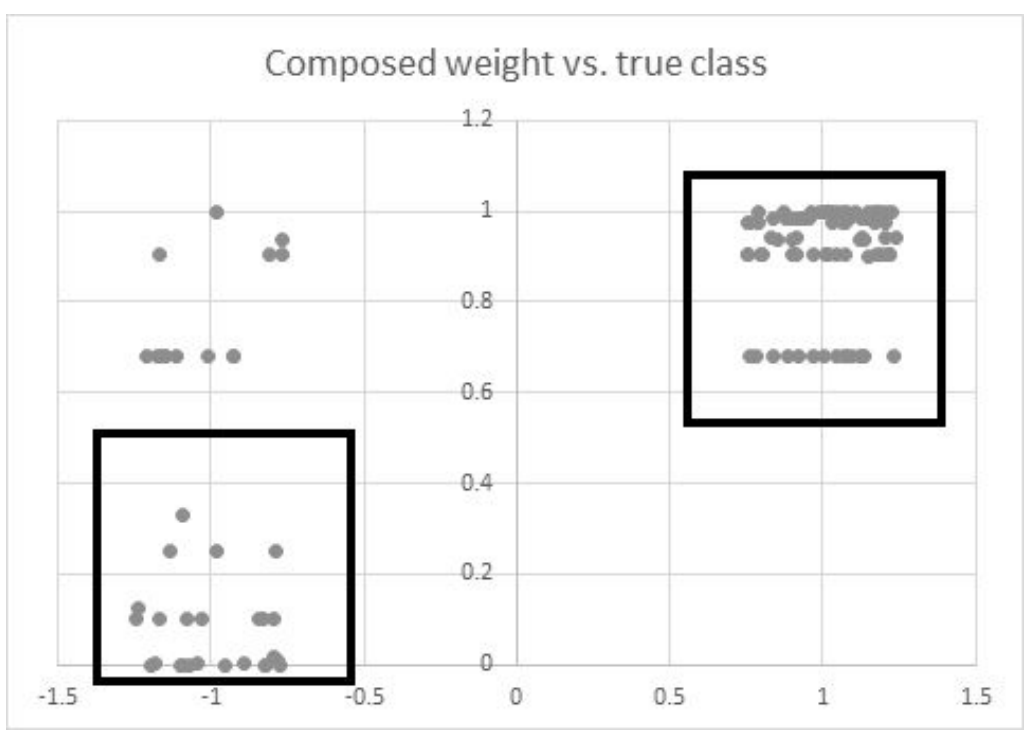

Fig. 10 Composed weights assigned to examples, KEX2 setting.

\section{Conclusions}

Credit risk assessment, credit scoring or loan applications approval are typical tasks that can be performed using machine learning or data mining techniques. From this viewpoint, loan applications evaluation is a classification or concept-description task, in which the learned knowledge is inferred from data about past decisions. 
Rule-learning algorithms are easier for humans to understand, when compared, e.g., with regression models, neural networks or SVM-created models.

This paper presents two data mining procedures implemented in the LISp-Miner system (4ft-Miner and KEX) and shows how these procedures can be used for the credit risk assessment task. 4ft-Miner is primary intended for association rules mining. Because each part of the rule is generated separately, $4 \mathrm{ft}-$ Miner can easily be used for concept description; here the succedent remains unchanged and must be set to the category defining the concept. KEX creates a set of rules that can be used directly for classification.

Our aim was to check the feasibility of both procedures for the given task by comparing them with the state-of-the-art rule-learning algorithms. We choose the PRISM, Jrip, PART and Ridor algorithms implemented in the Weka data mining system for this comparison. The empirical comparison is based on four data sets from the loan application domain. The results of experiments reported in Section 4 show that there is no single best algorithm that would outperform the other algorithms. KEX, PRISM, Jrip and Ridor each became the winning algorithm at least once when using the criterion based on the weighted sum of ranks achieved for accuracy and size of the rule set. 4ft-Miner cannot be used in this comparison as the resulting rules are not directly used for classification. We can thus compare the result of $4 \mathrm{ft}-$ Miner with the other algorithms only in terms of the size of the rule set. Here we can see from Tab. V and Tab. VI that 4ft-Miner usually creates more rules than the other algorithms. 4ft-Miner thus describes the concept in more ways and helps to gain broader insight into the knowledge hidden in the data.

We can also compare the algorithms themselves on the basis of their analysis. Here we can see that PART, PRISM, Jrip and Ridor all follow the set covering principle. That is, examples covered by a rule are removed from the training data. The consequence of this principle is that just one rule is applicable when classifying an example. This makes the classification process very simple (scan the rule set to find an applicable rule), but narrows the way the class is described. On the contrary, 4ft-Miner and KEX, repeatedly go through the whole training set when creating the rules. This results in the fact, that more rules can cover an example. For 4ft-Miner it means that partially overlapping rules can be obtained (as illustrated on the rules 07 and 10 in Tab. 4). The consequence for KEX is in the necessity to consider a higher number of applicable rules during classification. The solution for this problem is described in Section 3.2; KEX uses a compositional approach inspired by uncertainty processing in early expert systems. Another difference between KEX and the other rule-learning algorithms is that KEX does not perform crisp yes/no classification but assigns weights to the classes. This, together with the possibility to tune resulting decision strategy (in our case the final loan approval decision based on the classification result by setting the value of parameter $\alpha$ ) brings an extra advantage over a simple yes/no decision.

To summarize the results of the evaluation and comparison, $4 \mathrm{ft}$-Miner is well suited for the concept description task offering alternative viewpoints on identical examples belonging to the target concept and KEX is well suited for creating decision support systems that do not take over too much responsibility from the human users (which can be a crucial requirement in the loan application domain). 


\section{Acknowledgement}

This paper was prepared with the contribution of long-term institutional support of research activities by the Faculty of Informatics and Statistics, University of Economics, Prague.

\section{References}

[1] AGRAWAL R., IMIELINSKI T., SAWAMI A. Mining associations between sets of items in massive databases. In: Proc. of the ACM-SIGMOD Int. Conference on Management of Data, Washington D.C. 1993, pp. 207-216, doi: 10.1145/170036.170072.

[2] BERKA P. Workshop Notes on Discovery Challenge, Prague, Univ. of Economics, 1999.

[3] BERKA P. Learning compositional decision rules using the KEX algorithm. Intelligent Data Analysis. 2012, 16(4), pp. 665-681, doi: 10.3233/IDA-2012-0543.

[4] BRUHA I., BERKA P. Empirical Comparison of Various Discretization Procedures. Int.J. of Pattern recognition and Artificial Intelligence. 1998, 12(7), pp. 1017-1032, doi: 10.1142/ S0218001498000567.

[5] CENDROWSKA J. PRISM: An algorithm for inducing modular rules. Int. J. of ManMachine Studies. 1987, 27(4), pp. 349-370, doi: 10.1016/s0020-7373(87)80003-2.

[6] CHAPMAN P., CLINTON J., KERBER R., KHABAZA T., REINARTZ T. SHEARER C. WIRTH R. CRISP-DM 1.0 Step-by-step data mining guide. 2000, SPSS Inc.

[7] CHEN W., XIANG G., LIU Y., WANG K. Credit risk Evaluation by hybrid data mining technique. Systems Engineering Procedia. 2012, 3, pp. 194-200, doi: 10.1016/j. sepro. 2011. 10.029 .

[8] COHEN W. Fast Effective Rule Induction. In: 12th International Conference on Machine Learning, Tahoe City, California. 1995, pp. 115-123, doi: 10.1016/b978-1-55860-377-6. 50023-2.

[9] DUDA R.O., GASCHING J.E., HART P. Model Design in the Prospector Consultant System for Mineral Exploration. In: WEBER, NILSSON ed., Readings in Artificial Intelligence. Elsevier, 1981, doi: 10.1016/B978-0-934613-03-3.50028-3.

[10] FAYYAD U., IRANI K. Multi-interval discretization of continuous-valued attributes for classification learning, In: Proc. 13th Joint Conf. of Artificial Intelligence (IJCAI'93), 1993, pp. $1022-1027$.

[11] FRANK E., WITTEN I.H. Generating Accurate Rule Sets Without Global Optimization, In: Fifteenth International Conference on Machine Learning. 1998, pp. 144-151.

[12] GAINES B.R., COMPTON P. Induction of Ripple-Down Rules Applied to Modeling Large Databases. J. Intell. Inf. Syst. 1995, 5(3), pp. 211-228, doi: 10.1007/BF00962234.

[13] GALINDO J., TAMAYO P. Credit Risk Assessment using Statistical and Machine Learning: Basic Methodology and Risk Modeling Applications. Computational Economics. 2000, 15(12), pp. 107-143.

[14] HÁJEK P. Combining Functions for Certainty Factors in Consulting Systems. Int. J. ManMachine Studies. 1985, 22, pp. 59-76, doi: 10.1016/S0020-7373(85)80077-8.

[15] HÁJEK P., HAVRÁNEK T. Mechanising Hypothesis Formation - Mathematical Foundations for a General Theory. Springer, 1978, doi: 10.1007/978-3-642-66943-9.

[16] HALL M., FRANK E., HOLMES H., PFAHRINGER B., REUTWMANN P., WITTEN I. The WEKA Data Mining Software: An Update. SIGKDD Explorations. 2009, 11(1), doi: $10.1145 / 1656274.1656278$

[17] KERBER R. ChiMerge: Discretization of Numeric Attributes. In: Proc. AAAI-92 conference. AAAI Press, 1992, pp. 123-128.

[18] KIM K.S., HWANG H.J. An Integrated Data Mining Model for Customer Credit Evaluation. In: Proc. Int. Conf. Computational Science and Its Applications ICCSA, Singapore. Springer, 2005, s.vol. 3482, pp. 798-805, doi: 10.1007/11424857_87. 


\section{Neural Network World 5/2016, 497-518}

[19] KOTSIANTIS S. Credit risk analysis using a hybrid data mining model. Int. J. Intelligent Systems Technologies and Applications. 2007, 2(4), pp. 345-356, doi: 10.1504/ijista. 2007. 014030.

[20] LEE T.S., CHIU CH., CHOU Y.CH., LU CH. Mining the customer credit using classification and regression tree and multivariate adaptive regression splines. Computational Statistics 83 Data Analysis. 2006, 50(4), pp. 1113-1130, doi: 10.1016/j.csda.2004.11.006.

[21] LEE C., SHIN D. A context-sensitive discretization of numeric attributes for classification learning. In: COHN ed., 11th European Conf. on Artificial Intelligence (ECAI'94). John Wiley, 1994, pp. 428-432.

[22] QUINLAN J.R. Induction of decision trees. Machine Learning. 1986, 1(1), pp. 81-106, doi: $10.1007 / \mathrm{BF} 00116251$.

[23] QUINLAN J.R. Simplifying decision trees. Int J Man-Machine Studies. 1987, 27, pp. 221234, doi: 10.1016/S0020-7373(87)80053-6.

[24] QUINLAN J.R. Learning logical definitions from relations. Machine Learning. 1990, 5, pp. 239-266, doi: $10.1007 / \mathrm{BF} 00117105$.

[25] QUINLAN J.R. C4.5: Programs for machine learning. Morgan Kaufman, 1993.

[26] RAS Z., WIECZORKOWSKA A. Action-Rules: How to Increase Profit of a Company. In: ZIGHED, KOMOROWSKI, ZYTKOW, eds. Principles of Data Mining and Knowledge Discovery. Springer, 2000, pp. 587-592, doi: 10.1007/3-540-45372-5_70.

[27] RAUCH J. Observational Calculi and Association Rules. Springer, 2013, doi: 10.1007/ 978-3-642-11737-4.

[28] RAUCH J., ŠIMŮNEK M. Dobývání znalostí z databází, LISp-Miner a GUHA. Oeconomia Praha, 2014. In Czech.

[29] ŠIMŮNEK M. Academic KDD Project LISp-Miner. In: ABRAHAM, FRANKE, KOPPEN, eds. Advances in Soft Computing Intelligent Systems Design and Applications. SpringerVerlag, 2003, pp. 263-272, doi: 10.1007/978-3-540-44999-7_25.

[30] VINCIOTTI V., HAND D.J. Scorecard construction with unbalanced class sizes. Journal of the Iranian Statistical Society. 2003, 2(2), pp. 189-205.

[31] WOLPERT D.H. The lack of a priori distinctions between learning algorithms. Neural computation. 1996, 8(7), pp. 1341-1390, doi: 10.1162/neco.1996.8.7.1341.

[32] ZHOU L., WANG W. Loan Default Prediction on Large Imbalanced Data Using Random Forests. TELKOMNIKA Indonesian Journal of Electrical Engineering. 2012, 10(6), pp. 1519-1525, doi: 10.11591/telkomnika.v10i6.1323. 